Vietnam Journal of Mechanics, VAST Vol. 26, 2005, No.4 (208-214)

\title{
ON A FORM OF LYAPUNOV EXPONENTS (I: ESTABLISHMENT OF THE FORM)
}

\author{
NGUYEN VAN DINH \\ Institute of Mechanics
}

\begin{abstract}
Another form of Lyapunov exponents associated with certain motion that given by (1.2) is proposed. It is expressed through the variation of the disturbance direction (not through that of the disturbance norm).
\end{abstract}

\section{Introduction}

It is known that Lyapunov exponents associated with certain motion $X(t)$ (equilibrium state, periodic and quasi periodic or chaotic motions) are real numbers characterizing the behaviour of nearby motions $x(t)$ and provide informations on the stability character of the motion under consideration. In [1], they are defined as asymptotic quantities

$$
\lambda\left(y_{0}\right)=\lim _{t \rightarrow \infty} \frac{1}{t} \ln (\|y(t)\| /\|y(0)\|),
$$

where $\|\quad\|$ denotes a vector norm, $y(t)=x(t)-X(t)$ is the disturbance i.e. the deviation from the reference motion $X(t)$ to its nearby ones $x(t), y_{0}=y(0)$ is the initial disturbance, In stands for the natural logarithm. In the present paper, an attention is foccussed on another form of Lyapunov exponents, that is

$$
\lambda\left(u_{0}\right)=\lim _{t \rightarrow \infty} \frac{1}{t} \int_{0}^{t} u^{\prime}(\tau) A(\tau) u(\tau) d \tau,
$$

where $u(t)=y(t) /\|y(t)\|$ is the unit vector directed to the disturbance, $u_{0}=u(0)$ is the initial unit vector, $A(t)$ is the Jacobian matrix of the equation of variation, prime denotes the transposes operator.

In this part I, the form (1.2) is established; the part II is devoted to the verification and illustration of the results obtained.

\section{The equation of variation and the expression of the dis- turbance}

Consider a system governed by the differential equation

$$
\dot{x}(t)=F(x, t) \quad \text { or } \quad \dot{x}_{j}(t)=F_{j}\left(x_{1}, x_{2}, \ldots, x_{n}, t\right), \quad(j=1,2, \ldots, n),
$$


where $\mathbf{x}\left(x_{1}, x_{2}, \ldots, x_{n}\right)$ is the column vector in an $n$-dimensional Euclidean space with norm $\|\mathbf{x}\|=\sqrt{x_{1}^{2}+x_{2}^{2}+\cdots+x_{n}^{2}}$, overdot denotes differentiation with respect to time $t$, $F(x, t)$ is a column vector function (with necessary properties).

Let $X(t)$ be a motion of interest (the reference motion) - a solution of (2.1)

$$
\dot{X}(t)=F(X(t), t) \quad \text { or } \quad \dot{X}_{j}=F_{j}\left(\left(X_{1}(t), X_{2}(t), \ldots, X_{n}(t), t\right) \quad(j=1,2, \ldots, n) .\right.
$$

Imposing on $X(t)$ a disturbance $y(t)$, the corresponding nearby motion $x(t)$ can be expressed as:

$$
x(t)=X(t)+y(t) \quad \text { or } \quad x_{j}(t)=X_{j}(t)+y_{j}(t) \quad(j=1,2, \ldots, n) .
$$

Substituting (2.3) into (2.1), expanding $F(X(t)+y(t), t)$ in a Taylor series about $X(t)$, on account of (2.2) and retaining only linear terms in $y(t)$ leads to the equation of variation

$$
\dot{y}(t)=A(t) y(t) \quad \text { or } \quad \dot{y}_{j}(t)=\sum_{k=1}^{n} A_{j k}(t) y_{k}(t), \quad(j=1,2, \ldots, n),
$$

where $A(t)=\left[A_{j k}(t)\right]$ is the Jacobian matrix-an $n \times n$ matrix with elements

$$
A_{j k}(t)=\partial F_{j}\left(X_{1}(t), X_{2}(t), \ldots, X_{n}(t), t\right) / \partial x_{k}, \quad(j, k=1,2, \ldots, n) .
$$

Let $y(t)$ be a solution of the equation of variation and $\lambda$ is its Lyapunov exponent (one among Lyapunov exponents associated with $X(t)$ ). For simplicity, we use as definition the following expression (which differs from that corresponding in [2] by the sign minus):

$$
\lim _{t \rightarrow \infty} \frac{1}{t} e^{-\lambda t}\|y(t)\|=0
$$

Therefore, if $y(t)$ is written in the form

$$
y(t)=e^{\lambda t} \varphi(t) \quad \text { with } \varphi(t)=e^{-\lambda t} y(t),
$$

we have

$$
\lim _{t \rightarrow \infty} \frac{1}{t} \ln (\|\varphi(t)\|)=0
$$

\section{A form of Lyapunov exponent}

In this section, the form (1.2) of Lyapunov exponents is established.

Substituting (2.7) into (2.4) yields:

$$
\lambda e^{\lambda t} \varphi(t)+e^{\lambda t} \dot{\varphi}(t)=A(t) e^{\lambda t} \varphi(t)
$$

or

$$
\lambda \varphi(t)=A(t) \varphi(t)-\dot{\varphi}(t) .
$$


Dividing two sides of (3.1) by $\|\varphi(t)\|$ then multiplying from the left by the unit vector

$$
u(t)=y(t) /\|y(t)\|
$$

we obtain

$$
u^{\prime}(t) \cdot \lambda \cdot \frac{\varphi(t)}{\|\varphi(t)\|}=u^{\prime}(t) A(t) \frac{\varphi(t)}{\|\varphi(t)\|}-\frac{\varphi^{\prime}(t) \dot{\varphi}(t)}{\|\varphi(t)\|^{2}}
$$

With regard that

$$
\begin{aligned}
& \frac{\varphi(t)}{\|\varphi(t)\|}=\frac{e^{-\lambda t} \varphi(t)}{\left\|e^{-\lambda t} \varphi(t)\right\|}=\frac{y(y)}{\|y(t)\|}=u(t) \\
& u^{\prime}(t) \cdot u(t)=\|u(t)\|^{2}=1, \quad u^{\prime}(t) A(t) \frac{\varphi(t)}{\|\varphi(t)\|}=u^{\prime}(t) A(t) u(t) \\
& \varphi^{\prime}(t) \dot{\varphi}(t)=\frac{1}{2}\left(\sum_{j=1}^{n} \varphi_{j}^{2}(t)\right)^{\bullet}=\frac{1}{2}\left(\|\varphi(t)\|^{2}\right)^{\bullet}
\end{aligned}
$$

from (3.3), it follows

$$
\lambda=u^{\prime}(t) A(t) u(t)-\frac{1}{2} \frac{\left(\|\varphi(t)\|^{2}\right)^{\bullet}}{\|\varphi(t)\|^{2}} .
$$

Integrating (3.5) from $t=0$ to $t$, then dividing by $t$ gives

$$
\begin{aligned}
\lambda & =\frac{1}{t} \int_{0}^{t} u^{\prime}(\tau) A(\tau) u(\tau) d \tau-\frac{1}{2 t} \int_{0}^{t} \frac{d\left(\|\varphi(\tau)\|^{2}\right)}{\|\varphi(\tau)\|^{2}} \\
& =\frac{1}{t} \int_{0}^{t} u^{\prime}(\tau) A(\tau) u(\tau) d \tau-\left.\frac{1}{2 t} \ln \left(\|\varphi(\tau)\|^{2}\right)\right|_{0} ^{t} \\
& =\frac{1}{2 t} \int_{0}^{t} u^{\prime}(\tau) A(\tau) u(\tau) d \tau-\frac{1}{t} \ln (\|\varphi(t)\| /\|\varphi(0)\|) .
\end{aligned}
$$

At limit $(t \rightarrow \infty)$, on account of (2.8), we obtain (1.2).

$$
\lambda=\lim _{t \rightarrow \infty} \frac{1}{t} \int_{0}^{t} u^{\prime}(\tau) A(\tau) u(\tau) d \tau
$$

Remark. (1.2) can be deduced directly from (1.1). Indeed, for an interval $(0, T)$, examine the quantity

$$
\frac{1}{T} \ln (\|y(T)\| /\|y(0)\|)
$$


By dividing the interval $(0, T)$ into an infinity of infinitesimal interval of time $(t, t+d t)$, we transform (3.8) into $a$

$$
\begin{aligned}
\frac{1}{T} \ln \left(\frac{\|y(T)\|}{\|y(0)\|}\right) & =\frac{1}{T} \ln \left(\frac{\|y(d t)\|}{\|y(0)\|} \times \frac{\|y(2 d t)\|}{\|y(d t)\|} \times \cdots \times \frac{\|y(t+d t)\|}{\|y(t)\|} \times \cdots \times \frac{\|y(T)\|}{\|y(T-d t)\|}\right) \\
& =\frac{1}{T} \sum \ln (\|y(t+d t)\| /\|y(t)\|) .
\end{aligned}
$$

Using (2.4), we evaluate

$$
\begin{aligned}
\|y(t+d t)\| & =\|y(t)+A(t) y(t) d t\|=\left\{\sum_{j=1}^{n}\left(y_{j}(t)+\sum_{k=1}^{n} A_{j k}(t) y_{k}(t) d t\right)^{2}\right\}^{1 / 2} \\
& =\left\{\sum_{j=1}^{n}\left(y_{j}^{2}(t)+2 d t y_{j}(t) \sum_{k=1}^{n} A_{j k}(t) y_{k}(t)\right)\right\}^{1 / 2} \\
& =\|y(t)\|\left\{1+\frac{2 d t}{\|y(t)\|^{2}} y^{\prime}(t) A(t) y(t)\right\}^{1 / 2} \\
& =\|y(t)\|\left\{1+\frac{d t}{\|y(t)\|^{2}} y^{\prime}(t) A(t) y(t)\right\} .
\end{aligned}
$$

Thus

$$
\begin{aligned}
\ln \left(\frac{\|y(t+d t)\|}{\|y(t)\|}\right. & =\ln \left\{1+\frac{d t}{\|y(t)\|^{2}} y^{\prime}(t) A(t) y(t)\right\} \\
& =d t\left(\frac{y(t)}{\|y(t)\|}\right)^{\prime} A(t)\left(\frac{y(t)}{\|y(t)\|}\right)=d t \cdot u^{\prime}(t) A(t) u(t)
\end{aligned}
$$

and

$$
\frac{1}{T} \ln (\|y(T)\| /\|y(0)\|)=\frac{1}{T} \int_{0}^{T} u^{\prime}(t) A(t) u(t) d t .
$$

At limit $(T \rightarrow \infty)$ we obtain $(1.2)$

\section{The variation of the unit vector}

Obviously, to be able to use (1.2), we have to establish the differential equation which governes the variation of the unit vector $u(t)$.

Differentiating (3.2) with respect to $t$ yields

$$
\dot{u}(t)=\frac{\dot{y}(t)}{\|y(t)\|}-y(t) \cdot \frac{\|y(t)\|^{\bullet}}{\|y(t)\|^{2}} .
$$

Using (2.4), we calculate

$$
\frac{\dot{y}(t)}{\|y(t)\|}=A(t) \frac{y(t)}{\|y(t)\|}=A(t) u(t)
$$




$$
\begin{aligned}
& \|y(t)\|^{\bullet}=\frac{d}{d t}\left\{\sum_{j=1}^{n} y_{j}^{2}(t)\right\}^{1 / 2}=\frac{\sum_{j=1}^{n} y_{j}(t) \dot{y}_{j}(t)}{\|y(t)\|}=\frac{\sum_{j, k=1}^{n} A_{j k}(t) y_{j}(t) y_{k}(t)}{\|y(t)\|} \\
& y(t) \cdot \frac{\|y(t)\|^{\bullet}}{\|y(t)\|^{2}}=\frac{y(t)}{\|y(t)\|} A_{j k} \frac{y_{j}(t)}{\|y(t)\|} \cdot \frac{y_{k}}{\|y(t)\|}=u(t)\left(u^{\prime}(t) A(t) u(t)\right) .
\end{aligned}
$$

Hence, (4.1) becomes

$$
\dot{u}(t)=A(t) u(t)-u(t)\left\{u^{\prime}(t) A(t) u(t)\right\} .
$$

Introducing the notation

$$
\lambda(u)=u^{\prime}(t) A(t) u(t)
$$

we write (4.3) in the form

$$
\begin{aligned}
\dot{u}(t) & =A(t) u(t)-\lambda(u) \cdot u(t) \quad \text { or } \\
\dot{u}_{j}(t) & =\sum_{k=1}^{n} A_{j k}(t) u_{k}(t)-\lambda(u) u_{j}(t) \quad(j=1,2, \ldots, n) .
\end{aligned}
$$

Remark. By definition, each solution $y(t)$ of the equation of variation gives rise to one solutions $u(t)$ of the equation (4.5). However, from each solution $u(t)$ of (4.5), we obtain a family of solutions $y(t)$. Indeed, $y(t)$ can be found in the form:

$$
y(t)=\rho(t) \cdot u(t) .
$$

where $\rho(t)$ is a function to be determined.

Substituting (4.6) into (2.4), using (4.5) gives

$$
\dot{\rho} u+\rho \dot{u}=\cdot \rho t \rho[A u-\lambda(u) u]=A \rho(u),
$$

or

$$
\dot{\rho}(t)=\lambda(u)=u^{\prime}(t) A(t) u(t) .
$$

Hence

$$
\rho(t)=C e^{\left(u^{\prime}(t) A(t) u(t)\right) t}
$$

and

$$
y(t)=C e^{\lambda(u) t} \cdot u(t)
$$

where $C$ is an arbitrary constant.

\section{The case of an autonomous system}

An additional remark is related to autonomous system

$$
\dot{x}=F(x) \quad \text { or } \quad \dot{x}_{j}=F_{j}\left(x_{11}, x_{21}, \ldots, x_{n}\right), \quad(j=1,2, \ldots, n),
$$

where $F(x)$ does not depend explicitly on $t$. 
Let $X(t)$ be a "real" (not equilibrium state) and limited motion. As known, one of the Lyapunov exponents associated with $X(t)$ is equal to zero. Below, using (1.2), this property is verified.

Indeed, since the system is autonomous, if $X(t)$ is a solution, $X(t+h)=X(\tau)$ with arbitrary constant $h$ is a solution, too. Hence we have

$$
\dot{X}(\tau)=F(X(\tau)) \quad \text { or } \quad \dot{X}_{j}(\tau)=F_{j}\left(X_{1}(\tau), \ldots, X_{n}(\tau)\right), \quad(j=1,2, \ldots, n) .
$$

Differentiating (5.2) with respect to $t$ yields.

$$
\begin{aligned}
\ddot{X}_{j}(\tau) & =\left(\dot{X}_{j}(\tau)\right)^{\bullet}=\sum_{k=1}^{n}\left(\frac{\partial F_{j}\left(X_{1}(\tau), \ldots, X_{n}(\tau)\right)}{\partial x_{k}}\right) \dot{X}_{k}(\tau) \\
& =\sum_{k=1}^{n} A_{j k}(\tau) \dot{X}_{k}(\tau) \quad(j=1,2, \ldots, n) .
\end{aligned}
$$

The last equations mean that the derivative $\dot{X}(\tau)$ is a solution of the equation of variation (2.4). Let us calculate the Lyapunov exponent corresponding to this solution

$$
\lambda=\lim _{t \rightarrow \infty} \frac{1}{t} \int_{0}^{t} u^{\prime}(\tau) A(\tau) u(\tau) d \tau, \quad u(\tau)=\frac{\dot{X}(\tau)}{\|\dot{X}(\tau)\|} .
$$

Using (5.3), we have:

$$
\begin{aligned}
u^{\prime}(\tau) A(\tau) u(\tau) & =\left(\frac{\dot{X}}{\|\dot{X}\|}\right)^{\prime} A\left(\frac{\dot{X}}{\|\dot{X}\|}\right)=\frac{1}{\|\dot{X}\|^{2}} \sum_{j, k=1}^{n} A_{j k} \dot{X}_{j} \dot{X}_{k} \\
& =\frac{1}{\|\dot{X}\|^{2}} \sum_{j=1}^{n}\left(\sum_{k=1}^{n} A_{j k} \dot{X}_{k}\right) \dot{X}_{j}=\frac{1}{\|\dot{X}\|^{2}} \sum_{j=1}^{n} \ddot{X}_{j} \dot{X}_{j} \\
& =\frac{1}{2\|\dot{X}\|^{2}}\left(\sum_{j=1}^{n} \dot{X}_{j}^{2}\right)^{2}=\frac{1}{2} \frac{\left(\|\dot{X}\|^{2}\right)^{\bullet}}{\|\dot{X}\|^{2}}
\end{aligned}
$$

Hence

$$
\begin{aligned}
\lambda & =\lim _{t \infty} \int_{0}^{t} u^{\prime} A u d \tau=\lim _{t \rightarrow \infty} \frac{1}{2 t} \int_{0}^{t} \frac{d\|\dot{X}\|^{2}}{\|\dot{X}\|^{2}} \\
& =\left.\lim _{t \rightarrow \infty} \frac{1}{2 t} \ln \left(\|\dot{X}\|^{2}\right)\right|_{0} ^{t}=\lim _{t \rightarrow \infty} \frac{1}{t} \ln \left(\frac{\|\dot{X}\|}{\|\dot{X}(0)\|}\right)=0,
\end{aligned}
$$

since $\dot{X}(t)$ is limited.

\section{Comparison}

Let us make a comparison between (1.1) and (1.2). According to (1.1), Lyapunov exponents are connected with the variation of the norm of the disturbance. For numerical 
computation, (1.1) is replaced by a finite sum

$$
\lambda\left(y_{0}\right)=\frac{1}{T} \sum_{k=1}^{N} \ln \left(\frac{\|y(k T / N)\|}{\| y(k-1) T / N) \|}\right),
$$

where $T$-a chosen interval of time and $N$-a chosen positive integer must be large while $T / N$ must be very small.

Using (6.1) poses some difficulties. In (1.1), nearby motion $x(t)$ is assumed to be constantly close to the reference on $X(t)$ so that the disturbance $y(t)$ remains small from $t=0$ to $t=\infty$. In practice, especially for chaotic motion, it is not so: growing exponentially, $y(t)$ may becomes large even with small inttial $y(0)$ and even for small interval of time. A readjustment is thus necessary: as $y(t)$ becomes large enough, it is replaced by another one smaller in norm i.e. we must leave the initial nearby trajectory $x(t)$ and make a jump to another nearby one closer to the reference trajectory.

Contrarily, according to (1.2), only the variation of the direction of the disturbance $y(t)$ is of interest. This means that although Lyapunov exponents are the average rates of exponential expansion or contraction, they may be obtained only by examining the change of the disturbance direction. Imagine that we move just along the reference motion trajectory $X(t)$ (not along any nearby one), watching the change of the disturbance direction (not the variation of the norm of the disturbance): we are constantly on the reference trajectory, no jump is necessary. This fact may be useful.

\section{Conclusion}

The present article pays an attention on the form (1.2) of Lyapunov exponents. The establishment of this form is done, showing that Lyapunov exponents are connected with the variation of the disturbance direction.

This publication is completed with the financial support from The Council for Natural Science of Vietnam.

\section{References}

1. Ali H. Nayfeh, Balakumar Balachamdran, Applied Nonlinear Dynamics, John Willey, 1995.

2. Malkin I. G., Theory of Stability of Motion Nauka Moscow 1996.

Received December 5, 2003

\section{VỀ MÔT DANG CƯA SỐ MŨ LYAPUNOV}

Trong bài báo, một dạng của số mũ Liapunốp - dạng (1.2) được chú ý. Đã thiết lập dạng đó và kết quà cho thấy số mũ Liapunốp có liên quan chặt chẽ với sự biến đổi phương hướng của nhiễu. 